\title{
Airport Accessibility and Navigation Assistance for People with Visual Impairments
}

\author{
João Guerreiro \\ Carnegie Mellon University \\ jpvguerreiro@cmu.edu
}

\author{
Dragan Ahmetovic \\ Carnegie Mellon University \\ University of Turin \\ dragan.ahmetovic@unito.it
}

\author{
Daisuke Sato \\ Carnegie Mellon University \\ IBM Research \\ daisukes@cmu.edu
}

\author{
Kris Kitani \\ Carnegie Mellon University \\ kkitani@cs.cmu.edu
}

\author{
Chieko Asakawa \\ Carnegie Mellon University \\ IBM Research \\ chiekoa@cs.cmu.edu
}

\begin{abstract}
People with visual impairments often have to rely on the assistance of sighted guides in airports, which prevents them from having an independent travel experience. In order to learn about their perspectives on current airport accessibility, we conducted two focus groups that discussed their needs and experiences in-depth, as well as the potential role of assistive technologies. We found that independent navigation is a main challenge and severely impacts their overall experience. As a result, we equipped an airport with a Bluetooth Low Energy (BLE) beacon-based navigation system and performed a real-world study where users navigated routes relevant for their travel experience. We found that despite the challenging environment participants were able to complete their itinerary independently, presenting none to few navigation errors and reasonable timings. This study presents the first systematic evaluation posing BLE technology as a strong approach to increase the independence of visually impaired people in airports.
\end{abstract}

\section{CCS CONCEPTS}

- Human-centered computing $\rightarrow$ Empirical studies in accessibility; Accessibility technologies.

Permission to make digital or hard copies of all or part of this work for personal or classroom use is granted without fee provided that copies are not made or distributed for profit or commercial advantage and that copies bear this notice and the full citation on the first page. Copyrights for components of this work owned by others than the author(s) must be honored. Abstracting with credit is permitted. To copy otherwise, or republish, to post on servers or to redistribute to lists, requires prior specific permission and/or a fee. Request permissions from permissions@acm.org. CHI 2019, May 4-9, 2019, Glasgow, Scotland UK

(C) 2019 Copyright held by the owner/author(s). Publication rights licensed to ACM.

ACM ISBN 978-1-4503-5970-2/19/05 ..\$15.00

https://doi.org/10.1145/3290605.3300246
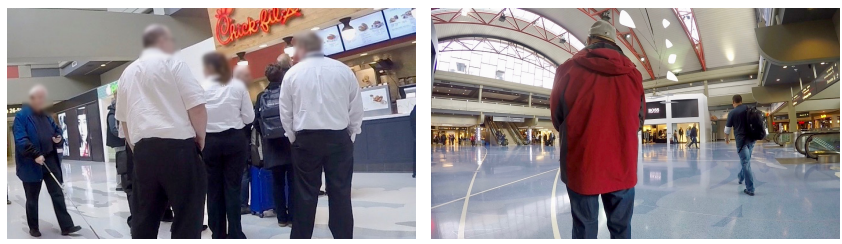

Crowd in a line, blocking the path

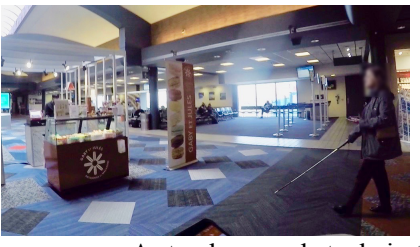

Large open space

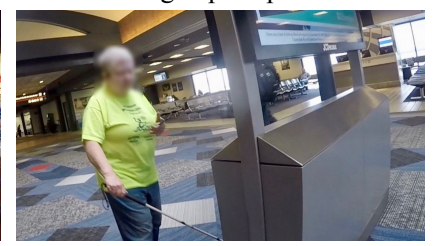

A stand or an obstacle in the middle of the corridor

Figure 1: Examples of challenges found by visually impaired participants navigating at the Pittsburgh International Airport using a smartphone-based navigation system.

\section{KEYWORDS}

Visual Impairment; blind navigation; orientation and mobility; airport accessibility; assistive technologies.

ACM Reference Format:

João Guerreiro, Dragan Ahmetovic, Daisuke Sato, Kris Kitani, and Chieko Asakawa. 2019. Airport Accessibility and Navigation Assistance for People with Visual Impairments. In CHI Conference on Human Factors in Computing Systems Proceedings (CHI 2019), May 4-9, 2019, Glasgow, Scotland UK. ACM, New York, NY, USA, 14 pages. https://doi.org/10.1145/3290605.3300246

\section{INTRODUCTION}

Despite recent efforts to increase airport accessibility [2], it is very challenging for people with visual impairments to have an independent air travel experience [38]. For that reason, they rely on the assistance of airport/airline personnel to get them from the ticketing counter to their gate. This service ensures their ability to travel, but presents several constraints when compared to sighted people's experience, 
such as longer waiting times and the inability to explore the airport in order to find restrooms, restaurants or shops [15].

Having a full understanding of such constraints is essential to design technologies able to improve airport accessibility. Yet, there is a lack of knowledge about the perspectives of visually impaired people concerning their travel experiences [38]. In this paper, we present two focus groups with visually impaired people aimed at discussing in-depth their needs, preferences, and the specific challenges they face in airports. We found that navigation within airports is particularly challenging, as is gaining knowledge about the environment. Moreover, participants reported a prominence of uncomfortable scenarios related to the inability to move independently in airports, in particular after being escorted to the gate.

Solutions to support indoor navigation have been implemented in airports, but there is a lack of systematic evaluations to assess the impact of such technologies [38]. Currently, several airports are equipped with location-based technologies aiming to revolutionize user's experience, including navigation assistance. Still, most efforts are focused on improving sighted people's experience, as the navigation does not consider the needs for higher accuracy and specific navigation instructions of people with visual impairments $[50,57,63]$. Although valuable exceptions target navigation for visually impaired people [7, 33, 37], there is very little knowledge about their implementations and an absence of known evaluations to understand their effectiveness.

To fill this gap, we implemented a state-of-the-art indoor navigation system for visually impaired people at the Pittsburgh International Airport (PIT), aimed at supporting independent mobility. We extended an open source project (HULOP [31]) by adapting a smartphone-based navigation app called NavCog [57], which uses Bluetooth Low Energy (BLE) beacons for highly accurate indoor localization. In order to assess its impact, we conducted a real-world user study at the airport where ten visually impaired users navigated four relevant routes for their air travel experience.

Results show that despite the difficulties of the environment, most users (six) traversed the routes without any navigation error while the others had very few. Error prevention techniques such as veering detection, and quickly re-routing when deviating from the path played a very important role in such result. This enabled most users to go across the airside terminal (310 meters) in less than 6 minutes. Moreover, their most relevant needs, finding the nearest restroom or going to a restaurant/shop while waiting at the gate, were generally achieved in approximately one and four minutes, respectively. This is the first systematic evaluation of a working indoor navigation system for people with visual impairments in airports and poses BLE technology (prevalent in airports nowadays, though for different purposes) as a strong approach to increase their independence.

\section{RELATED WORK}

People with visual impairments receive Orientation and Mobility training to learn how to travel safely and independently $[23,64]$. Still, this often falls short of supporting independent navigation, particularly in unfamiliar or complex locations $[24,65]$. A number of studies look into their experiences and the challenges they face in general mobility [10, 13, 42, 54], and there is significant work trying to improve public transportation accessibility [20, 29, 43]. However, experiences in airports are usually left out or addressed very briefly. Here, we describe prior work on the current status of airport accessibility, as well as in-situ blind navigation assistance.

\section{Airport Accessibility for Visually Impaired People}

Policies and laws against the discrimination of people with disabilities play a key role in increasing airport accessibility. For instance, the Airport Disability Compliance Program [3] tries to ensure that federal requirements are met by airports and airlines [2, 52]. It consists of training airport staff members, periodic evaluations and creating new resources. As a result, airports and airlines provide human assistants that escort people with disabilities to/from their gates. These services ensure a successful travel experience, but are sometimes accompanied with uncomfortable events [38,51], such as offering wheelchairs to visually impaired people, which is often seen as inappropriate or demeaning $[38,55,61]$.

When discussing assistive technologies in airports, navigation assistance (using BLE beacons) is presented as a promising approach to increase the independence of visually impaired people [38]. The number of airports equipped with BLE beacons keeps increasing, and standards for airport terminal beacons are already in practice [32]. Their goal is to improve the user experience by reducing waiting times, providing location-based deals or services, and supporting indoor navigation. However, the requirements for blind navigation assistance are different from the ones of sighted people. Although there are a few valuable exceptions that designed solutions for visually impaired people [7, 33, 37], very little is known about their implementation and effectiveness. A recent report to the Federal Aviation Administration (FAA) claims a lack of systematic studies that assess not only the current airport travel experience of visually impaired people, but also the impact of new assistive technologies [38].

\section{Indoor Navigation Assistance}

There are several GPS-based navigation systems for outdoor environments designed for visually impaired people $[8,22,34]$. Although widespread solutions are still not a reality indoors, there are several efforts to support indoor localization and blind navigation assistance [17, 57]. There is an increasing tendency for approaches where users do not 
require any hardware besides their own devices, for instance by making use of smartphone sensors [16, 21]. In addition, camera-based approaches can use the user's (or a specialized) device to guide them to a particular target $[4,18,41]$ or to detect and avoid obstacles [19, 39, 49, 62, 66].

Other approaches use sensor installations in the environment, such as Wi-Fi [11, 30], BLE beacons [40, 57], or both [36]. BLE technology is now a popular approach to support blind navigation and has been implemented in universities [47], shopping malls [57], airports [7, 33, 37], among others. For instance, NavCog provides turn-by-turn instructions, showing an average localization error below 1.65 meters [57]. Recent advances in Wi-Fi-based localization suggest it may soon catch up to BLE-based localization with the advantage of using existing infrastructure. Google recently announced [25] indoor localization support for Android 9 using the WiFi Round-Trip-Time (RTT) technique [12], while Apple Maps now supports Wi-Fi-based localization in several airports [56], including relevant Points-of-Interest (POIs) in their map. However, it does not support (blind) navigation assistance.

Independently of the technology, researchers are investigating how to better convey visual information and navigation instructions. Recent efforts include understanding what information is relevant $[44,50,63]$; how instructions should be conveyed $[46,59,63]$ and how users react to them $[35,48]$; the impact of different types of errors $[1,6]$; and how to convey information about people near the user [9]. In addition, Miao et al. [44] used the example of airports to collect requirements of indoor navigation systems; still, they do not focus on their experience in that environment. Finally, Spindler et al. [60] presented a navigation study conducted at an airport, but they used a Wizard-of-Oz approach to prompt navigation instructions instead of real-time localization.

In this paper, we increase the knowledge about the general experience of visually impaired people in airports, as well as their main challenges and coping mechanisms, by performing two exploratory focus groups. Then, we installed a navigation system at the Pittsburgh International Airport; and conducted a real-world study where visually impaired users navigated relevant routes at the airport.

\section{FOCUS GROUPS}

Based on our literature review on airport accessibility, we conducted two focus groups [26] with nine visually impaired participants. The goal of this exploratory research is to gain greater knowledge about the current status of airport accessibility, the main challenges faced by visitors with visual impairments, and the main requirements and opportunities to enhance their future travel experiences. The focus groups (five and four participants each) covered the same topics and each took approximately 90 minutes. The sessions were audio recorded and transcribed for further analysis.

\begin{tabular}{llllll} 
Part. & Studies & Age & Vision & $\begin{array}{l}\text { Primary } \\
\text { Aid }\end{array}$ & $\begin{array}{l}\text { AirTravel } \\
\text { p/ Year }\end{array}$ \\
\hline P1 & G1 + Nav & 70 & Blind & Cane & 1 to 3 \\
P2 & G1 + Nav & 65 & Blind & Cane & 1 to 3 \\
P3 & G1 + Nav & 62 & Blind & Dog & 1 to 3 \\
P4 & G1 & 33 & Blind & Dog & 1 to 3 \\
P5 & G1 & 46 & Blind & Dog & 1 to 3 \\
P6 & G2 + Nav & 43 & Blind & Cane & 1 to 3 \\
P7 & G2 & 72 & Blind & Cane & 1 to 3 \\
P8 & G2 & 76 & Blind & Cane & 1 to 3 \\
P9 & G2 + Nav & 70 & 20/200 & - & 1 to 3 \\
P10 & Nav & 42 & Blind & Dog & $<1$ \\
P11 & Nav & 37 & Blind & Dog & 4 to 6 \\
P12 & Nav & 40 & 20/400 & Cane & $<1$ \\
P13 & Nav & 54 & Blind & Dog & 4 to 6 \\
P14 & Nav & 69 & Blind & Cane & $<1$
\end{tabular}

Table 1: The participants of the focus groups (G1 or G2) and of the real-world navigation study (Nav).

\section{Participants}

We recruited nine participants $(4 \mathrm{~m} / 5 \mathrm{f})$, with ages ranging from 33 to $76(M=59.67, S D=15.17)$ years old. All participants were legally blind, but one had residual vision and walked without a navigation aid. There were four guide-dog and four white cane users. All participants visit airports at least once a year and were compensated $\$ 40$ for their time.

\section{Findings}

We performed a thematic analysis that resulted in six main themes that are described below.

The Lack of Independence in Airport Traveling. Most participants (except P3) are used to traveling by themselves in airports. For blind people, independence in an airport is seen as impossible, as illustrated by P5: "Independent traveling at the airport is pretty much impossible ... I am normally very independent, but when it comes to airports I kinda give up my right to be independent ... because it is so difficult!"

Such difficulties are not exclusive to totally blind people. P9 has residual vision and travels by himself, but still depends on the help provided by other pedestrians to orient himself in an airport. His inability to read the signs resulted in getting lost several times. Also, not using a navigation aid prompted rude or unhelpful comments from sighted people when he asks for help. The following comment from P9 led P7 and P8 to suggest using a white cane to prevent it: "A lot of people when I ask them for assistance ... they just look at me like 'What's wrong with you?' They point, 'like there's a monitor right up there'. And I've missed gates... so I ended up with a 5-hour layover that I shouldn't have had." 
Very rare exceptions may occur with frequent travelers that may learn a route at a particular airport. P2 used to travel frequently and would try to navigate to the gate by himself, relying on his (previous) guide dog to take him to relevant intersections. Yet, alike all participants, he had very little knowledge of his surroundings (e.g., restaurants, shops).

Personal Assistance Services. Personal assistance services are essential to guarantee that people with visual impairments are able to travel through an airport by taking them from the ticketing counter to their gate. When arriving at the check-in counter they are usually asked if they need assistance, which "takes generally less than 15 minutes, but not always." - (P1). Although reports usually focus on uncomfortable scenarios, participants' overall opinion about these services is positive, as they allow for an air travel experience with a good level of assistance. P6 stated that "Mostly, the level of assistance that I found, it's quite good. It's frequently good."

However, all participants were able to identify a number of limitations or uncomfortable experiences, which may affect their travel experience. Both focus groups started with wheelchair-related comments, an issue that most participants felt uncomfortable with: "I got a lot of 'Do you wanna go on a wheelchair?' A lot. It is very frustrating because ... I can walk." - (P4); "Even worse is when they bring one and they assume." - (P2). On the other hand, P7 had no concerns about using a wheelchair (". . specially if I got a really heavy backpack... Also, the general public is used to getting out of the way for wheelchairs and baggage carts ... Not necessarily for people”), while P6 saw both advantages and disadvantages ( "One reason is that they feel that they are able to move you around a little quicker than having you walking ... but it can be a little awkward and demeaning to sit on a wheelchair").

Other inconvenient episodes are related to language barriers in foreign (or even national) airports, or to losing control over their personal items (P6 and P7) when going through security or at the baggage claim (P2 got delivered a different bag). P7 stated: "You gotta be really really careful when you get out of security, because you totally lose control of everything you have, because they take it from you.... I had my autoharp and I said, do you have my green bag?... Well, when I get to the gate I don't have my autoharp."

Between and After Assistance. Participants agreed that the main limitations of their travel experiences occur after being escorted to the gate and when there are interruptions in the personal assistance services. The first is a major problem, especially when there are long layovers, delays or gate changes, as illustrated by P7: "My main problem at airports is that they are very good at getting you people to take you where you need to get to ... But when you get a 5 or 6 hour layover and you need to get something to eat and use the restrooms. that is a major hassle!. . It would be lovely to be able to get up and move around, and do things that you need to do, and maybe want to do!" In these situations, all blind participants mentioned they stay at the gate, as they do not know where to go and are afraid of not being able to come back.

In addition, waiting for personal assistance may result in distressing events when assistance fails to come, but also in a feeling of complete dependence on other people. P5 said: "You sit here, then somebody else will come pick you up, then you wait at another spot and someone will come. And in the meantime you really don't have option(s)... I feel like I am a piece of luggage! . . I am afraid of getting away, because things could change at any time, and will I be able to come back?... It's just too many possibilities of something that could go wrong, so I just sit and wait."

The Environmental Challenges of an Airport. Participants posed airports as very challenging environments for navigation. They outlined a set of reasons that make them particularly difficult, such as going through security, carrying luggage, very large open areas, and crowds either moving or stopped at particular locations: "I don't think it is very accessible, personally, due to how open it is. And people going every each way, and kinda like, New York City but in an airport ... And with luggage and all the sensory things going on, it can get overwhelming" - (P4). P1 reinforced this feeling: "I need help. But the thing that is really hard, is when people start moving and pushing around. ... and all of the sudden you can't keep track ... whether you're on a straight line."

Other prominent elements in most airports are escalators and moving walkways. In general, participants prefer to use escalators instead of elevators, because it is a quicker alternative. An exception is when they carry several bags or heavier luggage. Despite their preference, they still pointed out the challenges of finding the escalator that goes in the right direction, as pointed out by $\mathrm{P} 2$ : "My other favorite thing about airports is when you get to get on the escalator, and you don't realize you're on the wrong one until the cane is thrown back at you". Participants also had a positive opinion about moving walkways when going with someone, but not if they are traveling by themselves: "I like them, but I don't use them by myself. Just because I don't know how many breaks are gonna be ... If I'm going to find the next one" - (P1).

Travelers' Coping Mechanisms. When waiting at a gate the most common mechanism is to ask someone for assistance. P6 and P7 often ask their assistants to leave them next to the help desk. In other occasions they try to get assistance from nearby travelers, as illustrated by P2: "When mother nature calls and you really have to go, then I get up and just look lost and then somebody will say: Where are you going?"

Although in some occasions visually impaired travelers may need to ask for assistance to two or three people, in general other travelers are helpful and are able to help them 
with their current needs. P7 stressed that this is more difficult during long layovers when airports are less crowded: "The worst is when you are in a strange airport and it's late at night and there are no human beings around... and you've been sitting someplace for three hours and have another 2 hours and there is just nobody anywhere near you."

Another coping mechanism is to skip challenging steps, such as avoiding baggage claim by traveling only with carryon luggage (P4 and P8). P4 stated: "I try to do carry on as much as I can, because I don't really know how can I find my bag ... Going to the baggage claim can be very daunting ... And if there is one task I can eliminate, I'll try."

Opportunities for Assistive Technologies. When discussing how can technology enhance their overall experience in airports, some participants referred to gaining awareness of gate changes and delays, while others referred that airline smarphone apps support that. What stood out was all participants' need to gain greater awareness of their current location and what is around in order to gain more independence. Participants referred to older solutions such as Talking Signs [14] (proximity-based audio feedback), established parallels to mainstream approaches like GPS-based systems ("Something like a GPS, except indoors"-(P9)), and wondered if more recent approaches such as Aira [53] (camera-based remote assistance) would be valuable in an airport. P5 generalized by saying: "If I can always find where I am and where the bathroom is ... And ... what's around... If I can walk away from the waiting area ... If I have the confidence that I can come back to it. . . That would be wonderful."

P2 would also like to get more information about shops and restaurants (e.g., menus, existence of counter/tables). In addition, P3 referred to the potential value of knowing information beforehand ("If there is some kind of tactile model of the airport, like a tactile map. You get that picture in your head and then you come up into the center of the main area, you know that $A$ hall is to your left. . . You could kind of simulate through an app [P3 was familiar and mentioned both BlindSquare [8] simulation mode and NavCog Preview [27]].”).

\section{NAVIGATION ASSISTANCE AT THE AIRPORT}

The focus groups have reinforced the frequent need and desire of visually impaired travelers to move independently in airport environments. They are usually escorted to the gate area where they need to wait for boarding independently of how long it takes. Yet, they may need to go to the restroom or may want to eat or buy something at a nearby restaurant or shop. Occasionally, there may also be a gate change and the need to navigate to a different gate or terminal, but airports are particularly challenging environments to navigate.

To validate the ability of BLE-based technologies to support indoor navigation for visually impaired people in such a complex environment, we slightly adapted a mobile navigation app called NavCog, developed as an open source project (HULOP [31]). NavCog can provide real-time turn-by-turn instructions and alert about nearby Points-of-Interest (POIs). Moreover, it provides recovering instructions when users get off the planned route.

NavCog requires an annotated map [5] to define walkable areas and the location of POIs. We manually annotated the paths and static landmarks/POIs (e.g., obstacle, water fountain, floor change). POIs such as shops, restaurants or gates were automatically added using data from the airport map.

\section{Adaptations to NavCog}

Due to the additional challenges of airport environments, we extended the app to include the following:

Error prevention due to veering. This is specially relevant due to the very wide corridors and open areas found in airports. To prevent navigation errors, the system instructs the user to "bear right/left" when deviating from the planned path. It uses the user's location and orientation, the current walking speed, and the estimated localization accuracy. For instance, the threshold for veering prevention when the system is confident about its accuracy is a deviation of three meters, but this value increases when the system has lower confidence about the user's current position. Such measure avoids correcting users when they are on the planned route.

Moving Walkways. NavCog uses a pedestrian motion model combined with the beacon signals to compute the user's position. While this model improves localization accuracy when the user is walking, NavCog was not able to deal with moving walkways because the user was not moving. To solve this problem, we included heuristics to identify when the user enters the moving walkway. In that case, the system treats the motion model differently, so that it assumes movement when the user is stopped, and assumes greater velocity when the user is walking in the moving walkway.

\section{System Installation}

In collaboration with the Allegheny County Airport Authority, we installed 350 iBeacons at the Pittsburgh International Airport (PIT) in two days, which is currently covering approximately $33,000 \mathrm{~m}^{2}$ and includes the ticketing area, the train station (after security) and the path from the train to the central area of the airside terminals, the central area itself, and one of the four terminal corridors (Figure 2). We did not install beacons near the security area. The iBeacons were placed in about 10 to 15 meters intervals, except in the large open space at the center of the terminal (where the beacons on both sides are separated by approximately 30 meters). The beacons were usually placed at a height between 2.5 and 3.5 meters, when possible on columns and 
on walls. We tried to ensure that the beacons were out of reach to airport visitors; and not too high (the airport has very high ceilings) as it would affect localization accuracy, but high enough so that beacon signals are less likely to be blocked by people, crowds or obstacles. We then conducted a site survey to collect fingerprints of iBeacons' radio wave signals for about 17.5 hours to be used as training data of the localization model; and an additional 2.1 hours to evaluate the accuracy of the localization model. Average localization error is 2.2 meters, and 4.5 meters at the 95 percentile using the method developed by Murata et al. [45].

Large open spaces negatively impact the localization model, since longer distances between beacons result in a lower localization accuracy [45]. In addition, changes in the environment may require additional efforts to install the system. For instance, there was a very large object for an event when we conducted the site survey, which was removed afterwards. Thus, we had to collect fingerprints again in that area. Another relevant issue concerns the moving walkway and how it divides a corridor in two. An error of 2-4 meters is acceptable in wide corridors, but localizing the user on the wrong side of the moving walkway may cause navigation errors.

\section{REAL-WORLD USER STUDY}

We performed a user study at the PIT airport, where our main goal was to investigate the effectiveness of smartphone-based navigation assistance for visually impaired people using BLE technology. We target navigation scenarios that comprise both the environmental challenges found in airports and the needs of blind travelers.

\section{Participants}

Ten participants with visual impairments $(6 \mathrm{~m} / 4 \mathrm{f})$, with ages ranging from 37 to $70(\mathrm{M}=55.2, \mathrm{SD}=13.57)$ years old, were recruited to participate in the study (Table 1). Five of them participated in the focus groups; all of them are legally blind, but two have residual vision (P9, P12). Four participants used a guide-dog, five used a white cane and P9 walked without a navigation aid. P6 only performed the first task, as he was not able to pass to the terminal area. Participants took approximately two hours to complete the study and were compensated for their time (\$25 per hour).

\section{Apparatus}

Participants used an iPhone 8 and the adapted NavCog app, which logged all events during the navigation tasks. Participants used their free hand to hold the smartphone and used AfterShokz bone-conductive headphones to receive the audio instructions without blocking the environmental sound. The experiment was video-recorded for further analysis.

\section{Routes}

We selected four routes based on the main challenges found in airports and on the potential scenarios for independent navigation. Participants performed the tasks in a fixed order, which resembles an actual travel experience. A randomized order would be impracticable due to security screening at the airport and not relevant in this context. The routes (Figure 2) comprise the following scenarios and challenges:

(1) From an entrance to a ticketing counter. This route is relevant even considering personal assistance services as they often start at the airline counter. It has 120 meters, four turns and seven POIs or landmarks. It has very wide corridors that can easily result in veering.

(2) From the train to the gate. The longest route has 310 meters, three turns and 26 POIs. It starts with two escalators from the first to the third floor. We included escalators, as it is generally not avoided by visually impaired people, but presents navigation challenges. Also, this route has a particularly large open area with 35 per 23 meters. It passes by a moving walkway, which causes a separation between each side of the concourse. This route intends to provide an alternative to the current need for personal assistance to the gate.

(3) From the gate to the nearest (male or female) restroom. This is naturally a very short route (30 to 40 meters) since most gates have restrooms nearby. We found relevant to include it due to the concerns showed by the focus group participants.

(4) From the gate to a restaurant. A 230-meter route that is shorter than Route 2, but has a greater density of POIs (30) and two turns. Again, it has very wide corridors, passes by the moving walkway and alike Route 2 passes by potentially crowded areas (e.g., gate areas and the food court). Both routes 3 and 4 intend to increase users' independence after being escorted to the gate, a main issue outlined during the focus groups.

\section{Procedure}

We obtained (IRB approved) informed consent from all participants beforehand. After participants arrived to the airport, we introduced our research, its goals and performed a demographics questionnaire. We explained how the navigation app works and clarified any doubt that participants might have had. Before every task, we stressed that the participant should focus on the navigation and on reaching the destination instead of exploring the environment. We also referred that participants should try to recover from errors by themselves and that the researchers would only be observers, but would always be nearby to guarantee their safety, intervening only when necessary. 


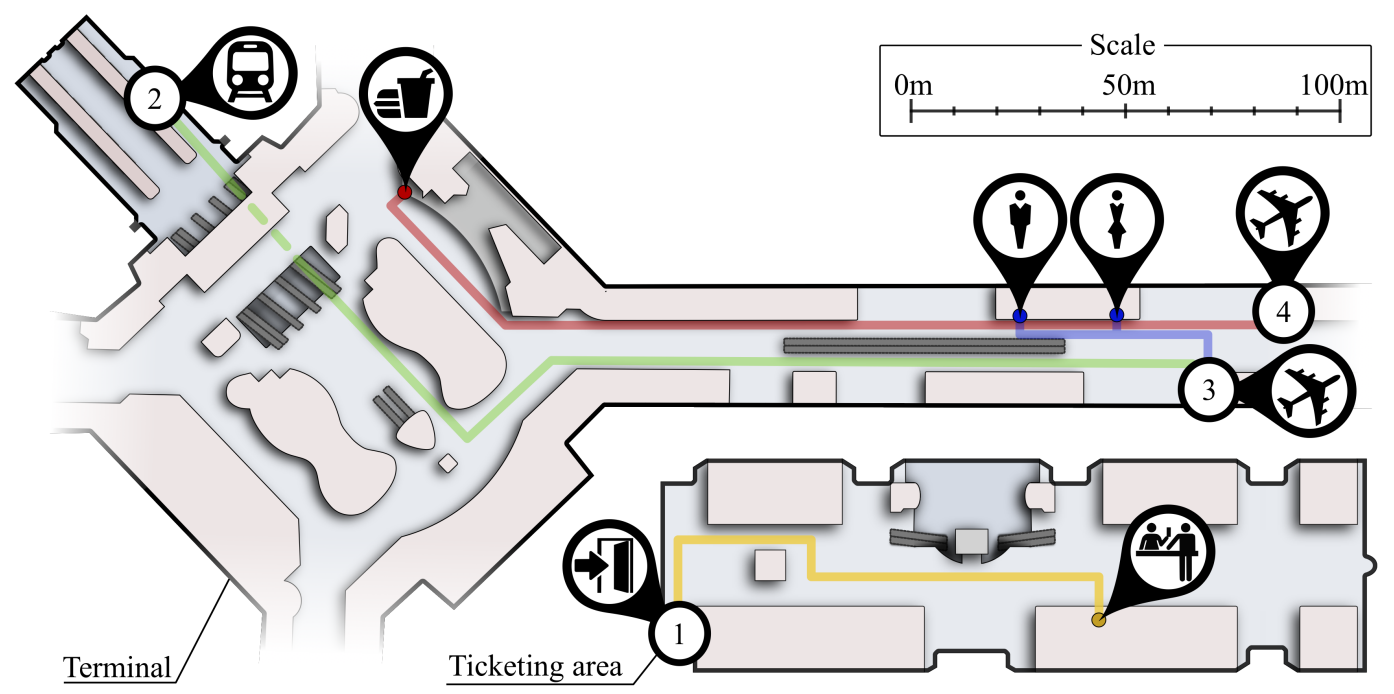

Figure 2: The experimental environment, which includes the ticketing area and the airside terminals. It presents the four routes used for the experiment. The numbers indicate each route starting point.

After performing Route 1 in the ticketing area, participants (and researchers) needed to go through the airport security screening and take the train to the terminals. Then, participants performed the three other routes sequentially. After completing each route, participants were asked to rate the easiness of the task (using the single ease questionnaire [58]), their confidence while navigating that route (1- Not Confident at All to 7- Very Confident) and if they would use this app to navigate similar routes independently in their upcoming visits (1-Strongly Disagree to 7-Strongly Agree).

After finishing all routes, we performed a post-interview to understand the users' perspectives on the effectiveness of the navigation app in this environment, persisting challenges and future directions to improve airport accessibility.

\section{RESULTS}

Our main goals were to analyze the ability of an accurate navigation app to support independent mobility of visually impaired travelers in airport environments. We report user performance based on completion times and errors, and perform an in-depth analysis based on video observations complemented by users' subjective feedback.

\section{Overall Performance}

All participants completed all routes, reaching the destination successfully (except P6 who only performed Route 1). Participants completed each task, on average, in 189 ( $\mathrm{SD}=56.6$ ), $418(\mathrm{SD}=147), 57.8(\mathrm{SD}=7.9)$, and $276(\mathrm{SD}=73.6)$ seconds, respectively (Figure 3). The difference in timings is justified by the length and complexity of the four routes. Their average walking speed was $0.86 \mathrm{~m} / \mathrm{s}(\mathrm{SD}=0.22)$, and the average walking speed of participants with/without guide dog was $1.01 \mathrm{~m} / \mathrm{s}(\mathrm{SD}=0.21)$ and $0.73 \mathrm{~m} / \mathrm{s}(\mathrm{SD}=0.12)$ respectively.

Six participants (P9-P13 and P6) completed the study without any navigation error. In general, there were one and six navigation errors in Routes 1 and 2 (the longer and most complex route), respectively. Of those, in three of them users passed right next to the escalator (Figure 6), while the other four errors were caused by a (system) orientation error after re-localizing users. This occurred only for the first participants: as it was detected and fixed by the researchers. However, this error caused confusion as users seemed lost until the system recovered or researchers intervened. We intervened four additional times to prevent any inconvenient situation although there was a chance that participants would recover by themselves. These events include getting very close to an off-route escalator (going down); entering stores, or almost bumping into a family near the dining area.

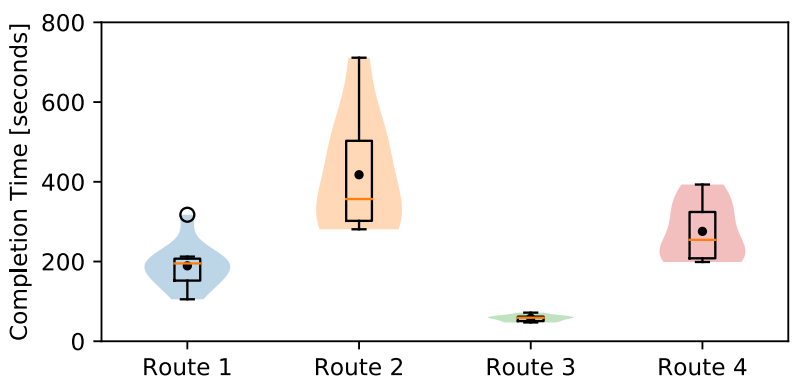

Figure 3: Completion time per route. Boxes show 25 and 75 percentiles, and medians. Black dots show means. 


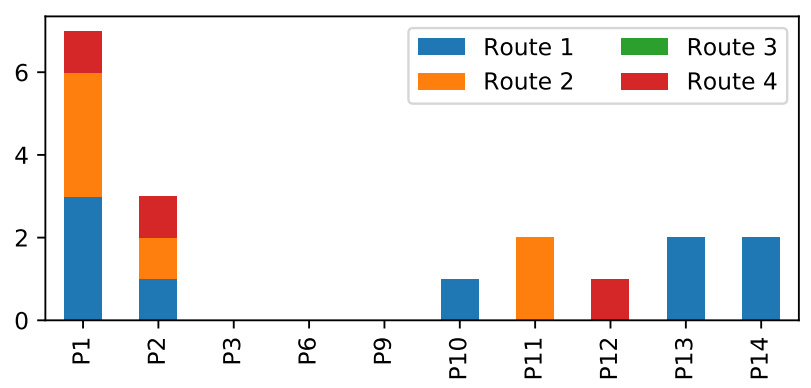

Figure 4: Number of times the system detected that the participant needs to veer in wide corridors.

\section{Video-Observation Analysis}

By observing the navigation tasks, we performed an in-depth analysis to understand the main challenges of this environment and what behaviours influenced users' performance.

Veering was common, but often corrected. All routes included open areas or very wide corridors. Such environments are known to be challenging for visually impaired people (particularly white-cane users) since there are more chances for users to veer and deviate from their path [18, 28]. It is important to note that we do not consider veering as a navigation error, unless it takes the user to an erroneous path. Participants used their navigation skills when possible to cope with those challenges; for instance, P2 noticed that along part of Routes 2 and 4 there was a difference in floor type in the same direction that he was heading; therefore, he followed a straight line on the intersection of the tile and carpet floors.

All participants except P3 (a guide dog user), P6 (did only one route) and P9 (who has residual vision) veered at least one time, but were usually able to recover by themselves. Figure 4 shows the number of times that the system provided error prevention instructions ("bear right/left") to maintain the user on the correct path (the values for P1 are affected by the aforementioned orientation errors). For instance, P2 and P11 veered in Route 2 in the large open area after the escalator, but were corrected with a "bear left" message that helped them proceeding on the intended path (Figure 5).

Another common location for veering was in in the connecting area between the center area and the concourse corridor (Route 2). While the system planned a route on the right-hand side of the concourse, five users veered after the 45-degree turn, ending up on the left-hand side of the moving walkway. Based on their location when re-routed, P1 and P14 continued their path on the left-hand side of the moving walkway and turned to the right side only when approaching the gate. On the other hand, P2, P11 and P12 were re-routed earlier and therefore took the right-hand side of the moving walkway. Interestingly, P11 ended up intentionally using the moving walkway when alerted about its presence.

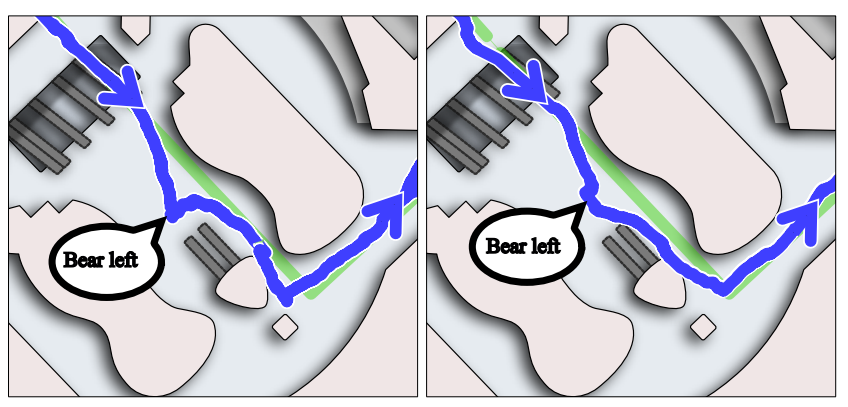

Figure 5: It shows the planned route (in green) and the walking trajectory (in blue) of P2 (L) and P11 (R) for Route 2.

While veering is often corrected with messages to adjust the orientation or by re-routing, the system does not intervene when there is a small deviation from the path due to uncertainty. This happens because the threshold for veering detection is based on the current estimate of localization accuracy and the perceived user location and orientation. While in most occasions users ended up correcting themselves using their navigation skills, there were very few occasions where the researcher needed to intervene due to safety concerns. For instance, in Route 1 P10 was only instructed to bear right when he was very close to an escalator going down. Although the system ended up providing such instruction, it was too close to a potentially dangerous situation (hence the intervention). This suggests that alternative solutions should be explored in areas that involve greater risk. In addition, P1 veered in the area depicted in Figure 5 but to the left side, leading her to enter a store without being corrected.

The challenge of finding the escalator. Six participants went straight to the escalator in Route 2, having no problems to find it. On the other hand, P2, P3 and P14 veered slightly to the right passing by the escalator just by a few meters. P2, a white-cane user, recovered by himself when he noticed that he passed by the escalator, by tapping on the screen to know what he should do next. P3, a guide-dog user, continued to walk forward (Figure 6) to an area that is not covered by the system. As the researchers intervened and asked to restart the task, P3 requested to be guided right in front of the escalator in order to teach the dog a command to find the escalator. In this case, the guide dog avoided the escalator because she was unfamiliar with it, suggesting that elements that are less common in their daily lives may result in navigation errors. After being taught and restarting the route the guide dog went straight to the escalator.

The impact of navigation aids, pedestrians and crowds. It is well known that guide dogs ease the navigation of people with visual impairments as they are able to avoid obstacles and to follow open paths when directed by their owner. In 


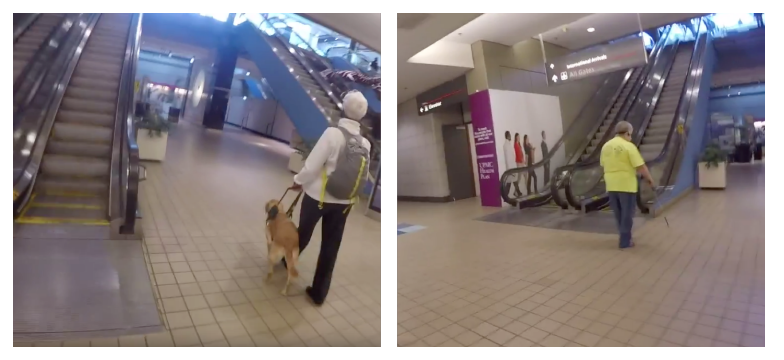

Figure 6: P3 (left) and P14 (right) veering near the escalator.

general, it results in faster navigation and less errors, even though sometimes the dog may take the lead and cause navigation errors [28], as shown by the escalator example. In airports, it is common to see crowds of pedestrians moving (e.g., coming from or moving to the gates) or stationary (e.g., in lines near the gates or in the food court), which can block part of the user's path. While guide dogs could often find their way among crowds, in several occasions sighted people eased that process by clearing the path either for guide dog or white cane users. When that did not happen, white cane users took longer to recover, but were generally able to resume navigation in the correct orientation. An exception occurred when P12 was interrupted by a person asking if he needed help, which led him to resume navigation in a slightly different orientation. This led to a re-route near the moving walkway (Route 2), but did not cause an error.

Interaction-based mechanisms for confirmation. During navigation, user-system interaction is mainly a one-way communication channel where the system guides the user to a destination. However, users could always tap on the screen to know what to do next. In fact, this command was used by all but one participant (P6) both in situations where they found something unexpected (e.g., P1 used it when finding a wall or almost entering a shop), but also as confirmation. For instance, P13 (who completed the study without navigation errors) used this command eight times as a confirmation that he was in the correct path. Another mechanism to ensure a correct orientation was used when performing slight turns, which are reported to be more challenging [6]. P10 and P13 stated that they performed the slight turns slower than regular turns in order to get the confirmation sound and vibration when reaching the correct orientation.

\section{Subjective Feedback}

Quantitative Scores. After each task, we collected users' subjective feedback to assess the perceived ease in performing the task, confidence and willingness to use the system to traverse similar routes in the future. While all routes were found to be easy, route 2 presented some difficulties. The other routes had an average score of 6.14 out of $7(\mathrm{SD}=0.96)$, route 2 scored $5.67(\mathrm{SD}=1.22)$. Specifically, 6 participants reported difficulties in taking the escalator, which may be the cause for the lower score. Participants' confidence built up steadily as they experienced more routes, starting from an average of $6.3(\mathrm{SD}=0.67)$ for Route 1 , and reaching $6.8(\mathrm{SD}$ $=0.44$ ) for Route 4 . The desire to independently experience similar routes increased even faster. From a score of 6.5 (SD $=0.53$ ) in Route 1, it received a full score from all participants by Route 3. This shows participants' drive to be independent in airports, particularly when they are at the gate.

After the tasks, we also collected general feedback on the system. Participants considered the system to be accurate, with an average score of 6.78 out of $7(\mathrm{SD}=0.44)$ and easy to use $(M=6.78 ; \mathrm{SD}=0.44)$. Thus, participants reported that they would use the system during their airport visits $(M=$ $6.89, \mathrm{SD}=0.33)$. The information provided by the system was also found to be complete $(M=6.89 ; \mathrm{SD}=0.33)$. Only two participants requested additional information: $P 2$ suggested to add trashcans as POIs, possibly allowing to activate and deactivate specific POIs as needed; and P11 proposed to provide more detailed information on the escalators, such as their direction, which is difficult to understand non visually.

When comparing the perceived difficulty to use escalators, we found no significant differences between navigating them with $(\mathrm{M}=3.11, \mathrm{SD}=1.90)$ and without $(\mathrm{M}=4.11, \mathrm{SD}=2.15)$ a navigation system. In contrast, other challenging areas were perceived to be less difficult to traverse with guidance: open areas difficulty significantly decreased $(p=.006)$ from a score of $6.33(\mathrm{SD}=0.87)$ without to $3(\mathrm{SD}=2)$ with guidance; difficulty to traverse crowded areas significantly decreased $(p=.04)$ from $5.44(\mathrm{SD}=1.67)$ without to $4(\mathrm{SD}=1.58)$ with guidance. The self-reported confidence in navigating through the airport improved significantly with the navigation app $(p=.0003)$, from a score of $3(\mathrm{SD}=1.80)$ to $6.89(\mathrm{SD}=0.33)$.

Qualitative Feedback. The overall feedback about using the navigation app at the airport was very positive. One reason is its availability in a public space often visited by the participants ("Seeing it here, it kind of blows me away! It is really cool!" - P12). Another reason is the ability to gain contextually-relevant knowledge about the environment, which is not available when being escorted by the airport personnel. While in the focus groups participants referred to an inability to find restaurants or shops independently, they were not aware of the choices available at the airport ("I didn't know that all of this was here!" - P2).

As users familiarized with the app, they also started developing coping mechanisms to improve their navigation performance. For instance, P12 who has residual vision would often turn before the system instruct him to turn. This behavior could sometimes cause an erroneous instruction (e.g., telling the user to turn, even though he is already with the correct 
orientation). To cope with this, P12 avoided "getting ahead of it [the system] ... Give it a chance to catch up with the user speed". Another example came from P2, who claimed that he started by depending exclusively on the navigation app, but then he learned "to rely more on my [his] senses and use NavCog as an aid, so I [he] started walking more confidently".

When asked about challenges, participants often referred to the escalator. First, it may be difficult to find when the user is further away. Second, they may "hear it when it's close, but not its direction" - P11. Also, participants (P1, P9, P3, and $\mathrm{P} 10)$, mentioned that holding the phone is uncomfortable in situations such as getting on the escalator. Although it is possible to use the system with the smartphone in the pocket or by wearing a strap, such usage negatively impacts localization accuracy. Future work should explore how to integrate data (during the site survey) that also represent this type of usage, and study how to minimize the impact of bodybased signal blocking. An additional challenge mentioned by P14 was the noise, in particular in crowded areas. The inability to hear a command, may be the reason for users tapping on the screen to listen the current instruction.

Suggestions for improvements include: obtaining more information about POIs ('stop somewhere and know more about it" - P1; being able to go through security (or ease that process); and customization for distance announcements. Finally, although P10 found he would navigate with the app at the airport by himself, he felt the need for other safety measures and referred to the importance of having a ('button to ask for help", in case anything goes wrong.

\section{DISCUSSION}

In this section, we discuss the main findings of the focus groups and of the real-world navigation study at the airport.

\section{Perspectives of People with Visual Impairments}

The focus groups shed light on the opinions and concerns that visually impaired people have about the accessibility of airports. In the end, the complexity of the environment leads them to "give up my [their] right to be independent... because it is so difficult!" The assistance provided by sighted guides is what ensures their ability to get to their boarding gate.

Although participants referred to the variability in the accessibility of different airports, all of them experienced uncomfortable episodes worldwide. For instance, alike previous reports [38, 55, 61], participants mentioned that wheelchairs are often not presented to visually impaired people in a proper and dignifying way. However, P7 has a contrasting opinion as she believed it to be acceptable, in particular when carrying heavy luggage. Such uncomfortable episodes show that greater efforts should be put in establishing guidelines for the interaction with people with disabilities, and in training the personnel that are providing assistance. On the other hand, reports about experiences in airports are usually related to negative comments or events. Yet, we learned that in general participants were satisfied with such services, being more concerned about the time they spend by themselves.

At the Gate. The main limitations that visually impaired people face when traveling by themselves occur when they are already at the gate. After being escorted to their gate they have no option but to wait, independently of the waiting times, delays or gate changes. Such limitations go beyond needing to wait for longer times, even when their flight is on time [15]. When they have to find a restroom or their new gate, they have to ask someone or "just look lost and then somebody will say: Where are you going?" - (P2). This severely hinders their air travel experience, negating their ability to just "get up and move around" - (P7). For this reason, although participants can see advantages in using navigation systems in their overall airport experience, their most relevant scenario is to navigate from or to the gate.

\section{Independent Mobility using a Navigation App}

The routes used for the navigation tasks intended to cover potential scenarios for independent navigation of visually impaired travelers. They traversed the most challenging areas of this environment including large open spaces, escalators and areas with moving walkways. The navigation app used in this study enabled users to complete their tasks with none or few errors, showing also an ability to prevent them. As a result, most participants were able to reach a ticketing counter (120 meters) in roughly three minutes; traverse a terminal (310 meters) in roughly six minutes; go from the gate to the restroom (30-40 meters) in less than one minute; and go from the gate to a restaurant (230 meters) in roughly 4 minutes. These represent very satisfactory timings considering that on-demand assistance from sighted guides is not always possible and sometimes even unavailable [38]. Moreover, users valued gaining contextual knowledge about POIs that they would otherwise be unaware of.

Remaining Challenges. It is important to note that a fully independent air travel experience is still a challenge. For instance, going through security is challenging due to the security concerns themselves, large crowds and losing contact with the belongings. In addition, it is relevant to consider the confidence that visually impaired people have when traveling with a sighted assistant. Although users got more confident as they got familiar with the navigation app, it is unclear if they would navigate Route 2 by themselves, particularly with time constraints. On the other hand, users' performance and comments suggest that they would very likely navigate Routes 3 and 4 independently. While this can be seen as a starting point, we believe that greater exposure to the app and to the environment is likely to further increase users' performance and confidence [47]. 


\section{The Environmental Challenges of Airports}

The environmental challenges of airports can start with the system installation. For instance, very large open areas mean that the density of iBeacons in that area is much lower than usual, resulting in lower localization accuracy [45]. However, overall the average of 2.2 meters was enough for practical navigation assistance due to the airport wide corridors.

Good accuracy and error prevention mechanisms enabled the system to correct the users when deviating from the path. However, slightly veering off the path may not be detected by the system, in particular in locations with lower accuracy. This may be problem when the target path is narrow (e.g., getting on the escalator) or areas with greater risk (e.g., next to a store showcasing fragile items). In such cases, combining BLE-based technologies with other methods, such as camera-based approaches, may help preventing accidents. For instance, obstacle detection approaches could detect potentially dangerous or fragile elements and notify the user.

Other challenges of airports include temporary obstacles, such as crowds in front of a gate. While guide dogs are usually able to avoid people and find their way, it can be more challenging for white-cane users. In addition, (as mentioned by P4) crowds also move, which may disorient the user (or the dog) when there are alternative paths, and are noisy which may affect the ability to pick-up other sensory cues.

\section{The Impact of Individual Differences}

Both the quantitative metrics and the observation analysis showed an impact of users' visual abilities and choice of mobility aid in their navigation performance at the airport. Relevant findings include that guide dog users navigated faster, made less errors, and were able to more easily avoid obstacles and go through crowds (even though sometimes crowds can be distracting for the dog).

We also noticed that the requirements for high localization accuracy and veering detection are even more important for blind, white-cane users. The main reasons are that participants with low vision (this naturally depends on the level of residual vision and type of visual impairment) could identify an open path when walking on a straight corridor or when instructed to turn. The same happens for guide-dog users, where the dog can follow a straight path or find an open corridor when instructed to turn by their owner. In contrast, blind, white-cane users can more easily deviate from the intended path due to a slight early/late instruction or by slightly overshooting a turn. While such deviation could often be corrected either by the user's own mobility skills (e.g., sensing a wall or a change in floor type leading them to readjust their orientation) or by the system (by re-routing or providing veering correction instructions), in some occasions a slight deviation also resulted in navigation errors or problematic situations (e.g., entering a store, or almost bumping into a family in the food court).

We have recruited a diverse set of participants, which includes guide-dog and white-cane users, as well as people who are blind and people with low vision (with and without a navigation aid). However, a higher number of participants, in particular with low vision, would most likely provide additional insights to their needs, challenges and performance.

\section{CONCLUSION}

We presented the findings of two focus groups that illustrate the perceptions that people with visual impairments have regarding the accessibility of airports, which have been neglected in the literature [38]. These findings suggest that the main constraints experienced by visually impaired people in airports occur after being escorted to their gate. At this point, their lack of knowledge of the environment and their fear of getting lost leads to a single option: sitting and waiting. In order to empower them with more independence, we installed a BLE beacon-based navigation system (NavCog) at the Pittsburgh International Airport and analyzed its effect. Results of a study with ten visually impaired people showed that the system was able to cope with many navigation challenges of airports, such as users frequent veering in wide open areas. The low number of navigation errors and reasonable route completion times pose indoor navigation assistance as a promising tool to support independent mobility and to enhance the experience of visually impaired people in airports. However, a few navigation errors (or researcher's occasional need to intervene) also show that greater localization accuracy may be required when traversing areas that may present greater risks to the user's (or environment) safety.

To the best of our knowledge, this is the first formal evaluation of a working navigation system for visually impaired people at an airport. We note that most airports are already equipped or plan to equip their sites with BLE beacons. In addition, other localization methods (e.g., based on Wi-Fi) may become viable alternatives in the near future, easing the deployment and maintenance of apps like NavCog. Despite the availability of the required infrastructure, what is currently lacking is both the awareness and the support of the navigation needs of visually impaired travelers. We believe this study can be a valuable benchmark for new installations and for formal evaluation of navigation systems that are already in place $[7,33,37]$.

\section{ACKNOWLEDGMENTS}

We thank all participants, the Allegheny County Airport Authority, and the staff of the Pittsburgh International Airport. This work was sponsored in part by NSF NRI award (1637927), NIDILRR (90DPGE0003), Allegheny County Airport Authority, and Shimizu Corporation. 


\section{REFERENCES}

[1] Ali Abdolrahmani, William Easley, Michele Williams, Stacy Branham, and Amy Hurst. 2017. Embracing Errors: Examining How Context of Use Impacts Blind Individuals' Acceptance of Navigation Aid Errors. In Proceedings of the 2017 CHI Conference on Human Factors in Computing Systems (CHI '17). ACM, New York, NY, USA, 4158-4169. https:// doi.org/10.1145/3025453.3025528

[2] Federal Aviation Administration. 2017. AC 150/5360-14A - Access to Airports by Individuals with Disabilities. Retrieved in January, 2019 from https://www.faa.gov/documentLibrary/media/ Advisorycircular/150-5360-14A.pdf.

[3] Federal Aviation Administration. 2018. Airport Disability Compliance Program. Retrieved in January, 2019 from https://www.faa.gov/about/office ${ }_{\mathrm{o}} \mathrm{rg} / \mathrm{h}_{\text {headquarters }}$ ffices/acr/ com $_{\mathrm{c}} \mathrm{iv}_{\mathrm{s}}$ upport/disability ompliance/. $_{\mathrm{c}}$

[4] Dragan Ahmetovic, Cristian Bernareggi, Andrea Gerino, and Sergio Mascetti. 2014. Zebrarecognizer: Efficient and precise localization of pedestrian crossings. 2566-2571. https://doi.org/10.1109/ ICPR.2014.443

[5] Dragan Ahmetovic, Cole Gleason, Chengxiong Ruan, Kris Kitani, Hironobu Takagi, and Chieko Asakawa. 2016. NavCog: A Navigational Cognitive Assistant for the Blind. In Proceedings of the 18th International Conference on Human-Computer Interaction with Mobile Devices and Services (MobileHCI '16). ACM, New York, NY, USA, 90-99. https://doi.org/10.1145/2935334.2935361

[6] Dragan Ahmetovic, Uran Oh, Sergio Mascetti, and Chieko Asakawa. 2018. Turn Right: Analysis of Rotation Errors in Turn-by-Turn Navigation for Individuals with Visual Impairments. In Proceedings of the 20th International ACM SIGACCESS Conference on Computers and Accessibility (ASSETS '18). ACM, New York, NY, USA, 333-339. https://doi.org/10.1145/3234695.3236363

[7] Fraport TAV Antalya Terminal Isletmeciligi A.S. 2018. Antalya Airport SmartSteps. Retrieved in January, 2019 from https://appadvice.com/ game/app/antalya-airport-smart-steps/1246202175.

[8] BlindSquare. 2018. BlindSquare iOS Application. Retrieved in January, 2019 from http://blindsquare.com/.

[9] Stacy M. Branham, Ali Abdolrahmani, William Easley, Morgan Scheuerman, Erick Ronquillo, and Amy Hurst. 2017. "Is Someone There? Do They Have a Gun": How Visual Information About Others Can Improve Personal Safety Management for Blind Individuals. In Proceedings of the 19th International ACM SIGACCESS Conference on Computers and Accessibility (ASSETS '17). ACM, New York, NY, USA, 260-269. https://doi.org/10.1145/3132525.3132534

[10] Diana M Brouwer, Gaynor Sadlo, Karen Winding, and Marianne IG Hanneman. 2008. Limitations in mobility: experiences of visually impaired older people. British fournal of Occupational Therapy 71, 10 (2008), 414-421. https://doi.org/10.1177/030802260807101003

[11] Yu-Chung Cheng, Yatin Chawathe, Anthony LaMarca, and John Krumm. 2005. Accuracy Characterization for Metropolitan-scale WiFi Localization. In Proceedings of the 3rd International Conference on Mobile Systems, Applications, and Services (MobiSys '05). ACM, New York, NY, USA, 233-245. https://doi.org/10.1145/1067170.1067195

[12] Marc Ciurana, David López, and Francisco Barceló-Arroyo. 2009. SofTOA: Software ranging for TOA-based positioning of WLAN terminals. In International Symposium on Location-and Context-Awareness. Springer, 207-221. https://doi.org/10.1007/978-3-642-01721-6 3

[13] SM Cobb. 2012. National mobility report: Mobility experiences and perceptions of blind and vision impaired persons. Dublin, NCBI and Irish Guide Dogs for the blind (2012), 1-199.

[14] William Crandall, John Brabyn, Billie Louise Bentzen, and Linda Myers. 1999. Remote infrared signage evaluation for transit stations and intersections. Journal of Rehabilitation Research and Development 36, 4 (1999), 341-355.

[15] Simon Darcy. 2017. Accessibility as a key management component of the Paralympics. In Managing the Paralympics. Palgrave Macmillan, 49-92. https://doi.org/10.1057/978-1-137-43522-43

[16] Navid Fallah, Ilias Apostolopoulos, Kostas Bekris, and Eelke Folmer. 2012. The User As a Sensor: Navigating Users with Visual Impairments in Indoor Spaces Using Tactile Landmarks. In Proceedings of the SIGCHI Conference on Human Factors in Computing Systems (CHI '12). ACM, New York, NY, USA, 425-432. https://doi.org/10.1145/ 2207676.2207735

[17] Navid Fallah, Ilias Apostolopoulos, Kostas Bekris, and Eelke Folmer. 2013. Indoor human navigation systems: A survey. Interacting with Computers 25, 1 (2013), 21-33. https://doi.org/10.1093/iwc/iws010

[18] Alexander Fiannaca, Ilias Apostolopoulous, and Eelke Folmer. 2014. Headlock: A Wearable Navigation Aid That Helps Blind Cane Users Traverse Large Open Spaces. In Proceedings of the 16th International ACM SIGACCESS Conference on Computers \& Accessibility (ASSETS '14). ACM, New York, NY, USA, 19-26. https://doi.org/10.1145/ 2661334.2661453

[19] Vítor Filipe, Filipe Fernandes, Hugo Fernandes, António Sousa, Hugo Paredes, and João Barroso. 2012. Blind navigation support system based on Microsoft Kinect. Procedia Computer Science 14 (2012), 94101. https://doi.org/10.1016/j.procs.2012.10.011

[20] German Flores and Roberto Manduchi. 2016. Experiments with a public transit assistant for blind passengers. In International Conference on Computers Helping People with Special Needs. Springer, 43-50. https: //doi.org/10.1007/978-3-319-41267-27

[21] German Flores and Roberto Manduchi. 2018. Easy Return: An App for Indoor Backtracking Assistance. In Proceedings of the $2018 \mathrm{CHI}$ Conference on Human Factors in Computing Systems (CHI '18). ACM, New York, NY, USA, Article 17, 12 pages. https://doi.org/10.1145/ 3173574.3173591

[22] American Printing House for the Blind. 2018. Nearby Explorer. Retrieved in January, 2019 from http://www.aph.org/nearby-explorer/.

[23] Nicholas A Giudice. 2018. Navigating without Vision: Principles of Blind Spatial Cognition. In Handbook of Behavioral and Cognitive Geography. Edward Elgar Publishing, Chapter 15. https://doi.org/ 10.4337/9781784717544

[24] Nicholas A. Giudice and Gordon E. Legge. 2008. Blind Navigation and the Role of Technology. John Wiley \& Sons, Ltd, Chapter 25, 479-500. https://doi.org/10.1002/9780470379424.ch25

[25] Google. 2018. Introducing Android 9 Pie. Retrieved in January, 2019 from https://android-developers.googleblog.com/2018/08/ introducing-android-9-pie.html.

[26] Thomas L Greenbaum. 1998. The handbook for focus group research. Sage. https://doi.org/10.4135/9781412986151

[27] João Guerreiro, Dragan Ahmetovic, Kris M. Kitani, and Chieko Asakawa. 2017. Virtual Navigation for Blind People: Building Sequential Representations of the Real-World. In Proceedings of the 19th International ACM SIGACCESS Conference on Computers and Accessibility (ASSETS '17). ACM, New York, NY, USA, 280-289. https: //doi.org/10.1145/3132525.3132545

[28] João Guerreiro, Eshed Ohn-Bar, Dragan Ahmetovic, Kris Kitani, and Chieko Asakawa. 2018. How Context and User Behavior Affect Indoor Navigation Assistance for Blind People. In Proceedings of the Internet of Accessible Things (W4A '18). ACM, New York, NY, USA, Article 2, 4 pages. https://doi.org/10.1145/3192714.3192829

[29] Kotaro Hara, Shiri Azenkot, Megan Campbell, Cynthia L. Bennett, Vicki Le, Sean Pannella, Robert Moore, Kelly Minckler, Rochelle H. Ng, and Jon E. Froehlich. 2015. Improving Public Transit Accessibility for Blind Riders by Crowdsourcing Bus Stop Landmark Locations with 
Google Street View: An Extended Analysis. ACM Trans. Access. Comput. 6, 2, Article 5 (March 2015), 23 pages. https://doi.org/10.1145/2717513

[30] Suining He and S-H Gary Chan. 2016. Wi-Fi fingerprint-based indoor positioning: Recent advances and comparisons. IEEE Communications Surveys \& Tutorials 18, 1 (2016), 466-490. https://doi.org/10.1109/ COMST.2015.2464084

[31] HULOP. 2018. HULOP Human-scale Localization Platform - Github. Retrieved in January, 2019 from https://github.com/hulop/.

[32] Airports Council International. 2018. Airport Terminal Beacons Recommended Practice. Retrieved in January, 2019 from http: //www.aci.aero/media/6b4d6043-1bab-457c-8352-53c4c51dd634/ 5XBxhA/AboutACI/Priorities/IT-New/Initiativies/Beacons/ACIAirport-Terminal-Beacons-Recommended-Practice.pdf.

[33] Corinne Iozzio. 2014. Indoor mapping lets the blind navigate airports. https://www.smithsonianmag.com/innovation/indoormapping-lets-blind-navigate-airports-180952292/. (2014).

[34] Hernisa Kacorri, Sergio Mascetti, Andrea Gerino, Dragan Ahmetovic, Valeria Alampi, Hironobu Takagi, and Chieko Asakawa. 2018. Insights on Assistive Orientation and Mobility of People with Visual Impairment Based on Large-Scale Longitudinal Data. ACM Trans. Access. Comput. 11, 1, Article 5 (March 2018), 28 pages. https: //doi.org/10.1145/3178853

[35] Hernisa Kacorri, Eshed Ohn-Bar, Kris M. Kitani, and Chieko Asakawa. 2018. Environmental Factors in Indoor Navigation Based on RealWorld Trajectories of Blind Users. In Proceedings of the $2018 \mathrm{CHI}$ Conference on Human Factors in Computing Systems (CHI '18). ACM, New York, NY, USA, Article 56, 12 pages. https://doi.org/10.1145/ 3173574.3173630

[36] Loizos Kanaris, Akis Kokkinis, Antonio Liotta, and Stavros Stavrou. 2017. Fusing bluetooth beacon data with Wi-Fi radiomaps for improved indoor localization. Sensors 17, 4 (2017), 812. https://doi.org/10.3390/ s17040812

[37] Fredrick Kunkle. 2017. Blind travelers can get around an airport more easily with new app. Retrieved in January, 2019 from https: //www.washingtonpost.com/news/tripping/wp/2017/12/21/blindtravelers-can-get-around-an-airport-more-easily-with-new-app/.

[38] Gordon E Legge, Chris Downey, Nicholas A Giudice, and Bosco S Tjan. 2016. Indoor airport wayfinding for blind and visually impaired travelers. In Technical Report to the Federal Aviation Administration, No. DOT/Faa/TC-TN16/54. http://www.airporttech.tc.faa.gov/Download/Airport-SafetyPapers-Publications-Detail/dt/Detail/ItemID/572/Indoor-AirportWayfinding-for-Blind-and-Visually-Impaired-Travelers.

[39] Bing Li, J Pablo Munoz, Xuejian Rong, Jizhong Xiao, Yingli Tian, and Aries Arditi. 2016. ISANA: wearable context-aware indoor assistive navigation with obstacle avoidance for the blind. In Computer Vision, ECCV Workshops. Springer, 448-462. https://doi.org/10.1007/978-3319-48881-3 1

[40] Dierna Giovanni Luca and MachÃň Alberto. 2016. Towards accurate indoor localization using iBeacons, fingerprinting and particle filtering. In 2016 International Conference on Indoor Positioning and Indoor Navigation (IPIN).

[41] Roberto Manduchi and James M. Coughlan. 2014. The Last Meter: Blind Visual Guidance to a Target. In Proceedings of the SIGCHI Conference on Human Factors in Computing Systems (CHI '14). ACM, New York, NY, USA, 3113-3122. https://doi.org/10.1145/2556288.2557328

[42] Roberto Manduchi and Sri Kurniawan. 2011. Mobility-related accidents experienced by people with visual impairment. AER fournal: Research and Practice in Visual Impairment and Blindness 4, 2 (2011), 44-54.

[43] James R Marston and Reginald G Golledge. 2000. Towards an accessible city: Removing functional barriers for the blind and vision impaired: A case for auditory signs. (2000). Technical Report.
[44] Mei Miao, Martin Spindler, and Gerhard Weber. 2011. Requirements of indoor navigation system from blind users. In Symposium of the Austrian HCI and Usability Engineering Group. Springer, 673-679. https: //doi.org/10.1007/978-3-642-25364-548

[45] Masayuki Murata, Dragan Ahmetovic, Daisuke Sato, Hironobu Takagi, Kris M Kitani, and Chieko Asakawa. 2018. Smartphone-based Indoor Localization for Blind Navigation across Building Complexes. In Proceedings of the 16th IEEE International Conference on Pervasive Computing and Communications (PerCom '18). IEEE, 1-10. https: //doi.org/10.1109/PERCOM.2018.8444593

[46] Hugo Nicolau, Joaquim Jorge, and Tiago Guerreiro. 2009. Blobby: How to Guide a Blind Person. In CHI '09 Extended Abstracts on Human Factors in Computing Systems (CHI EA '09). ACM, New York, NY, USA, 3601-3606. https://doi.org/10.1145/1520340.1520541

[47] Eshed Ohn-Bar, João Guerreiro, Dragan Ahmetovic, Kris M. Kitani, and Chieko Asakawa. 2018. Modeling Expertise in Assistive Navigation Interfaces for Blind People. In 23rd International Conference on Intelligent User Interfaces (IUI '18). ACM, New York, NY, USA, 403-407. https://doi.org/10.1145/3172944.3173008

[48] Eshed Ohn-Bar, João Guerreiro, Kris Kitani, and Chieko Asakawa. 2018. Variability in Reactions to Instructional Guidance During SmartphoneBased Assisted Navigation of Blind Users. Proc. ACM Interact. Mob. Wearable Ubiquitous Technol. 2, 3, Article 131 (Sept. 2018), 25 pages. https://doi.org/10.1145/3264941

[49] En Peng, Patrick Peursum, Ling Li, and Svetha Venkatesh. 2010. A smartphone-based obstacle sensor for the visually impaired. In International Conference on Ubiquitous Intelligence and Computing. Springer, 590-604. https://doi.org/10.1007/978-3-642-16355-545

[50] J. Eduardo Pérez, Myriam Arrue, Masatomo Kobayashi, Hironobu Takagi, and Chieko Asakawa. 2017. Assessment of Semantic Taxonomies for Blind Indoor Navigation Based on a Shopping Center Use Case. In Proceedings of the 14th Web for All Conference on The Future of Accessible Work (W4A '17). ACM, New York, NY, USA, Article 19, 4 pages. https://doi.org/10.1145/3058555.3058575

[51] Yaniv Poria, Arie Reichel, and Yael Brandt. 2010. The flight experiences of people with disabilities: an exploratory study. fournal of Travel Research 49, 2 (2010), 216-227. https://doi.org/10.1177/ 0047287509336477

[52] Supriya Raman. 2011. Airport accessibility for travellers with disabilities. Fournal of Airport Management 5, 3 (2011), 239-244.

[53] Dan Reed. 2018. A.I. 'Glasses' Turn Airports, Other Chaotic Places For The Blind Into Liberating Experiences. Retrieved in January, 2019 from https://www.forbes.com/sites/danielreed/2018/02/06/ a-i-glasses-turn-airports-other-chaotic-places-for-the-blind-intoliberating-experiences.

[54] Abbas Riazi, Fatemeh Riazi, Rezvan Yoosfi, and Fatemeh Bahmeei. 2016. Outdoor difficulties experienced by a group of visually impaired Iranian people. Journal of current ophthalmology 28, 2 (2016), 85-90. https://doi.org/10.1016/j.joco.2016.04.002

[55] Victoria Richards, Annette Pritchard, and Nigel Morgan. 2010. (Re) Envisioning tourism and visual impairment. Annals of Tourism Research 37, 4 (2010), 1097-1116. https://doi.org/10.1016/j. annals.2010.04.011

[56] Rene Ritchie. 2018. Complete list of Apple Maps 'Look Inside' for Airports. Retrieved in January, 2019 from https://www.imore.com/ apple-maps-indoor-airports.

[57] Daisuke Sato, Uran Oh, Kakuya Naito, Hironobu Takagi, Kris Kitani, and Chieko Asakawa. 2017. NavCog3: An Evaluation of a SmartphoneBased Blind Indoor Navigation Assistant with Semantic Features in a Large-Scale Environment. In Proceedings of the 19th International ACM SIGACCESS Conference on Computers and Accessibility (ASSETS '17). ACM, New York, NY, USA, 270-279. https://doi.org/10.1145/ 3132525.3132535 
[58] Jeff Sauro and Joseph S. Dumas. 2009. Comparison of Three Onequestion, Post-task Usability Questionnaires. In Proceedings of the SIGCHI Conference on Human Factors in Computing Systems (CHI '09). ACM, New York, NY, USA, 1599-1608. https://doi.org/10.1145/ 1518701.1518946

[59] Morgan Klaus Scheuerman, William Easley, Ali Abdolrahmani, Amy Hurst, and Stacy Branham. 2017. Learning the Language: The Importance of Studying Written Directions in Designing Navigational Technologies for the Blind. In Proceedings of the 2017 CHI Conference Extended Abstracts on Human Factors in Computing Systems (CHI EA '17). ACM, New York, NY, USA, 2922-2928. https://doi.org/10.1145/ 3027063.3053260

[60] Martin Spindler, Michael Weber, Denise Prescher, Mei Miao, Gerhard Weber, and Georgios Ioannidis. 2012. Translating floor plans into directions. In International Conference on Computers for Handicapped Persons. Springer, 59-66. https://doi.org/10.1007/978-3-642-31534- 310

[61] Anja Thieme, Cynthia L. Bennett, Cecily Morrison, Edward Cutrell, and Alex S. Taylor. 2018. "I Can Do Everything but See!" - How People with Vision Impairments Negotiate Their Abilities in Social Contexts. In Proceedings of the 2018 CHI Conference on Human Factors in Computing Systems (CHI '18). ACM, New York, NY, USA, Article 203, 14 pages. https://doi.org/10.1145/3173574.3173777
[62] YingLi Tian, Xiaodong Yang, Chucai Yi, and Aries Arditi. 2013. Toward a computer vision-based wayfinding aid for blind persons to access unfamiliar indoor environments. Machine vision and applications 24, 3 (2013), 521-535. https://doi.org/10.1007/s00138-012-0431-7

[63] Wayfindr. 2017. Open Standard for audio-based wayfinding: Version 1.1. Retrieved in January, 2019 from https://www.wayfindr.net/wpcontent/uploads/2017/12/Wayfindr-Open-Standard-Rec-1.1.pdf.

[64] William R Wiener, Richard L Welsh, and Bruce B Blasch. 2010. Foundations of orientation and mobility. Vol. 1. American Foundation for the Blind.

[65] Michele A. Williams, Amy Hurst, and Shaun K. Kane. 2013. "Pray Before You Step out": Describing Personal and Situational Blind Navigation Behaviors. In Proceedings of the 15th International ACM SIGACCESS Conference on Computers and Accessibility (ASSETS '13). ACM, New York, NY, USA, Article 28, 8 pages. https://doi.org/10.1145/ 2513383.2513449

[66] Limin Zeng, Markus Simros, and Gerhard Weber. 2017. Camerabased Mobile Electronic Travel Aids Support for Cognitive Mapping of Unknown Spaces. In Proceedings of the 19th International Conference on Human-Computer Interaction with Mobile Devices and Services (MobileHCI '17). ACM, New York, NY, USA, Article 8, 10 pages. https://doi.org/10.1145/3098279.3098563 